\title{
Pandemic Productivity: Student-Run Free Clinic Integrates Behavioral Health in the Wake of COVID-19
}

\author{
Rebecca Fischbein ${ }^{1}$ (D) - Stacey Gardner-Buckshaw ${ }^{1} \cdot$ Ashley Loucek $^{1,2} \cdot$ Sairekha Ravichandran ${ }^{1} \cdot$ Madeleine Eicher $^{1}$. \\ John M. Boltri ${ }^{1}$
}

Received: 27 June 2020 / Accepted: 28 October 2020 / Published online: 17 November 2020

(C) Academic Psychiatry 2020

With the sudden onset of COVID-19, provision of medical and mental health services have rapidly evolved to incorporate virtual visits as an essential service [1-5]. Medical education also had to rapidly adapt to an online platform as students were displaced from clinical sites [6-10]. Our educational curriculum under development in a student-run free clinicintegration of behavioral health services including screening, brief intervention, and referral for treatment - had to be suddenly altered to accommodate displaced fourth-year medical students. This report describes the rapid adaptation of primary care in-person free clinic services to integrated, virtual, and telephone-based visits to (1) continue providing healthcare to underserved patients, (2) fulfill project goals for behavioral health integration, and (3) create meaningful clinical educational opportunities for students suddenly removed from clinical settings.

\section{Background}

Student-run free clinics are typically located in underserved areas and provide high quality, critical healthcare services free or at no cost for community members $[11,12]$. These clinics also provide authentic practice and leadership opportunities for student volunteers $[12,13]$. In addition to managing acute and chronic illness, many student-run free clinics have begun integrating behavioral health services with primary care [14-18]. Provision of behavioral health services is especially important among clinic patients who often have comorbid mental health needs [15]. Integration of behavioral health into

Rebecca Fischbein

rfischbein@neomed.edu

1 Northeast Ohio Medical University, Rootstown, OH, USA

2 Coleman Professional Services, Ravenna, OH, USA primary care-focused student-run free clinics increases medical student exposure to commonly treated behavioral health illnesses [17] and also matches national integration priorities [19].

The Northeast Ohio Medical University Student Outreach to Area Residents (SOAR) student-run free clinic was established in 2016 to provide free primary services to community members in Portage County, Ohio. Approximately $10 \%$ of working age Portage County adults are uninsured, $15 \%$ live in poverty, and about $17 \%$ adults failed to fill prescriptions from their doctor in the past year [20]. To address these community needs, SOAR is open three to four Saturdays a month with each clinic session staffed by approximately 22 students and 4 faculty volunteers. Since its start, over 242 unique patients have received free care, with a total of 722 patient visits including 996 free lab tests and 1418 prescriptions. In addition to primary care, services include immunizations, specialty care, social determinants of health screening and referral to community resources, pharmacy education, and disease management education.

A federal grant, received in June 2019, facilitated expansion of SOAR to increase primary care services, improve social equity in healthcare, develop and implement a competency-based telemedicine curriculum, and enhance provider wellness. Funding was also provided to develop, pilot test, and disseminate medical student and physician assistant student training programs that integrate behavioral health into multidisciplinary primary care teams. By the time COVID-19 emerged in March 2020, all first-year grant milestones were accomplished including a plan and timeline for implementation of telemedicine for primary care and behavioral health. The implementation team had begun researching: telehealth technology, competencies required for a telemedicine curriculum, electronic medical record options (the clinic had been using paper record), and behavioral health screening tools. We were on track for implementing behavioral health services in April 2020, 
an electronic medical record system during the summer, and piloting telemedicine curriculum in the fall.

However, as news of the COVID-19 pandemic spread, we realized there would be a significant negative impact on Northeast Ohio Medical University students, staff, and SOAR patients. During early March 2020, we discovered that over half of our fourth-year medical students, only 6 weeks from becoming physicians, would not be allowed to report to their final clerkship rotation sites. We also determined that SOAR patients would be at increased risk for adverse outcomes due to a combination of the pandemic, social vulnerability, and lack of access to primary services. We sought to address both problems by (1) keeping SOAR open and capitalizing on the work we had already began with regard to behavioral health and telemedicine integration and (2) providing SOAR-related clinical and experiential opportunities for displaced students.

Four SOAR clerkship opportunities were developed that addressed clinical competencies including patient care management, documentation, self-directed learning, and scholarship. Forty medical students were recruited and placed into teams focusing on integration of behavioral health, electronic medical records, telehealth technology and protocols, and social media campaigns. The present case report describes the experiences and work of students engaged in behavioral health integration and how their work coincided with the activities occurring in the telehealth technology team. This team was tasked with (1) selecting appropriate technology for telephone and virtual clinic visits, (2) designing related protocols, and (3) practicing and improving protocols.

\section{Description of the Elective}

The elective was a facilitated, independent learning rotation, designed and coordinated by three faculty with roles on the grant team: co-investigator, SOAR behavioral health consultant, and program evaluator. Input was also provided by the university's fourth-year clerkship director. Learning objectives for the course included (1) applying screening research conducted by the grant team to develop a SOAR behavioral health protocol, (2) utilizing quality improvement methods to assess the effectiveness of the protocol within the workflow and make real-time adjustments, (3) designing a training on the screening protocol and workflow for all SOAR volunteers, and (4) pilot testing and evaluating a training for SOAR volunteers. The Northeast Ohio Medical University Institutional Review Board determined that the evaluation of the training did not meet the definition of human subject research. Students were assessed on competencies related to patient care management, documentation, self-directed learning, scholarly activity, and presentation skills.
Students attended multiple virtual meetings each week for 4 weeks. On Monday and Friday mornings, all students in the SOAR electives attended a meeting that included assignments at the beginning of the week and student progress reports at the end of the week. Following the progress reports, students were given their assignments for SOAR Saturday patient care clinic. On Tuesday and Thursday mornings, the students focused on behavioral health integration engaged with the three faculty advisors for specific instructions and formative assessment on their work. On Saturdays, these students volunteered in the virtual SOAR clinic and worked with the behavioral health consultant to determine the best ways to implement behavioral health screening, intervention, and referral activities.

\section{Implementation}

\section{Week 1}

During week 1, the students reviewed behavioral health screening tools to be implemented in the clinic. For each screener, they compiled an annotated bibliography that examined (1) appropriateness for implementation in a primary care setting and (2) validity evidence, including estimates of sensitivity and specificity when available. The students also helped established decision rules for administration of the screeners (Table 1) and cut scores for referrals.

\section{Week 2}

In the second week of the elective, students were tasked with developing a behavioral health integration workflow, by incorporating the established patient care activities at SOAR with virtual clinic activities. Students attended the virtual clinic under the direction of the behavioral health consultant, trialed behavioral health screenings, and initiated interventions and referrals.

Prior to COVID-19, all SOAR patient visits were conducted in-person. With COVID-19, the telehealth technology team and faculty worked to quickly transition SOAR patient visits to virtual visits using a combination of video technology and cell phones. This included selection of technology and design of protocols. Individual video breakout rooms were created for each patient staffed by a senior (third- or fourth-year) medical student and a junior student. The senior student would call the patient via cell phone and obtain permission to put the cell phone on speaker so that the other student(s) could hear the patient through video/audio. When required, the student was able to return to the main video room to contact the preceptor and then bring the preceptor into the breakout room.

Also prior to COVID-19, we decided to pilot the administration of behavioral health screeners by mailing screeners to 
Table 1 Behavioral health screeners, related decision rules, and cut scores for referrals

\begin{tabular}{|c|c|c|c|}
\hline Screener & Description & Administration decision rule & Cut scores for referral \\
\hline $\begin{array}{l}\text { Patient Health } \\
\text { Questionnaire-2 (PHQ2) }\end{array}$ & Brief 2-item depression screen & $\begin{array}{l}\text { Every visit unless more in-depth } \\
\text { screening indicated }\end{array}$ & Score of 3 or higher \\
\hline $\begin{array}{l}\text { Patient Health } \\
\text { Questionnaire-4 (PHQ-4) }\end{array}$ & $\begin{array}{l}\text { Combined 4-item depression and anxiety } \\
\text { screen }\end{array}$ & $\begin{array}{l}\text { Each initial appointment to } \\
\text { establish a baseline }\end{array}$ & $\begin{array}{l}3 \text { or higher each section or total of } 6 \text { or } \\
\text { higher }\end{array}$ \\
\hline $\begin{array}{l}\text { Patient Health } \\
\text { Questionnaire-9 (PHQ-9) }\end{array}$ & $\begin{array}{l}\text { 9-item depression measure for screening, } \\
\text { diagnosing, monitoring severity of } \\
\text { depression }\end{array}$ & $\begin{array}{l}\text { Upon any identifiable risk or } \\
\text { presentation to clinic for } \\
\text { primary BH concern. }\end{array}$ & $\begin{array}{l}\text { Any score of } 10 \text { or more ( } 6 \text { for initial). } \\
\text { Any increase of } 3 \text { or more upon } \\
\text { subsequent screening }\end{array}$ \\
\hline $\begin{array}{l}\text { Primary Care } \\
\text { Post-Traumatic Stress } \\
\text { Disorder Screen } \\
\text { (PC-PSTD) }\end{array}$ & $\begin{array}{l}\text { 5-item measure designed for use in } \\
\text { primary care to identify and screen for } \\
\text { trauma }\end{array}$ & $\begin{array}{l}\text { If history of trauma reported or } \\
\text { symptoms of PTSD reported }\end{array}$ & Score of 3 or higher \\
\hline CAGE-AID & $\begin{array}{l}\text { 4-item screen used to screen for problems } \\
\text { related to alcohol or substance abuse } \\
\text { and dependence }\end{array}$ & $\begin{array}{l}\text { Initial appointment/establishing a } \\
\text { baseline }\end{array}$ & Score of 2 or higher \\
\hline $\begin{array}{l}\text { Prescription Opioid Misuse } \\
\text { Index (POMI) }\end{array}$ & $\begin{array}{l}\text { 6-item measure to identify patients who } \\
\text { misuse prescription opioids }\end{array}$ & $\begin{array}{l}\text { Patient prescribed opioids with } \\
\text { history of misuse or current } \\
\text { concerns of misuse }\end{array}$ & Score of 2 or higher \\
\hline
\end{tabular}

patients with instructions to bring completed screeners to their upcoming appointment. With the sudden change to a virtual clinic, we asked the fourth-year medical student team to work with the protocols established by the telehealth technology to integrate virtual screening into the clinic. They determined that the screenings should be conducted at the beginning of the appointment with the patient (see Fig. 1). Depending on the screener results, the behavioral health consultant was then brought in if needed.
The team piloted this process but quickly discovered the need to establish cut-off scores for referral to the behavioral health consultant (Table 1). This component was added to the workflow (see Fig. 1). Additionally, the student team and the behavioral health consultant discovered the need to better integrate the consultant in the screening process. Consequently, during the morning huddle, the behavioral health consultant would review the schedule for the day and pre-screen clients who have behavioral health history or are visiting for related

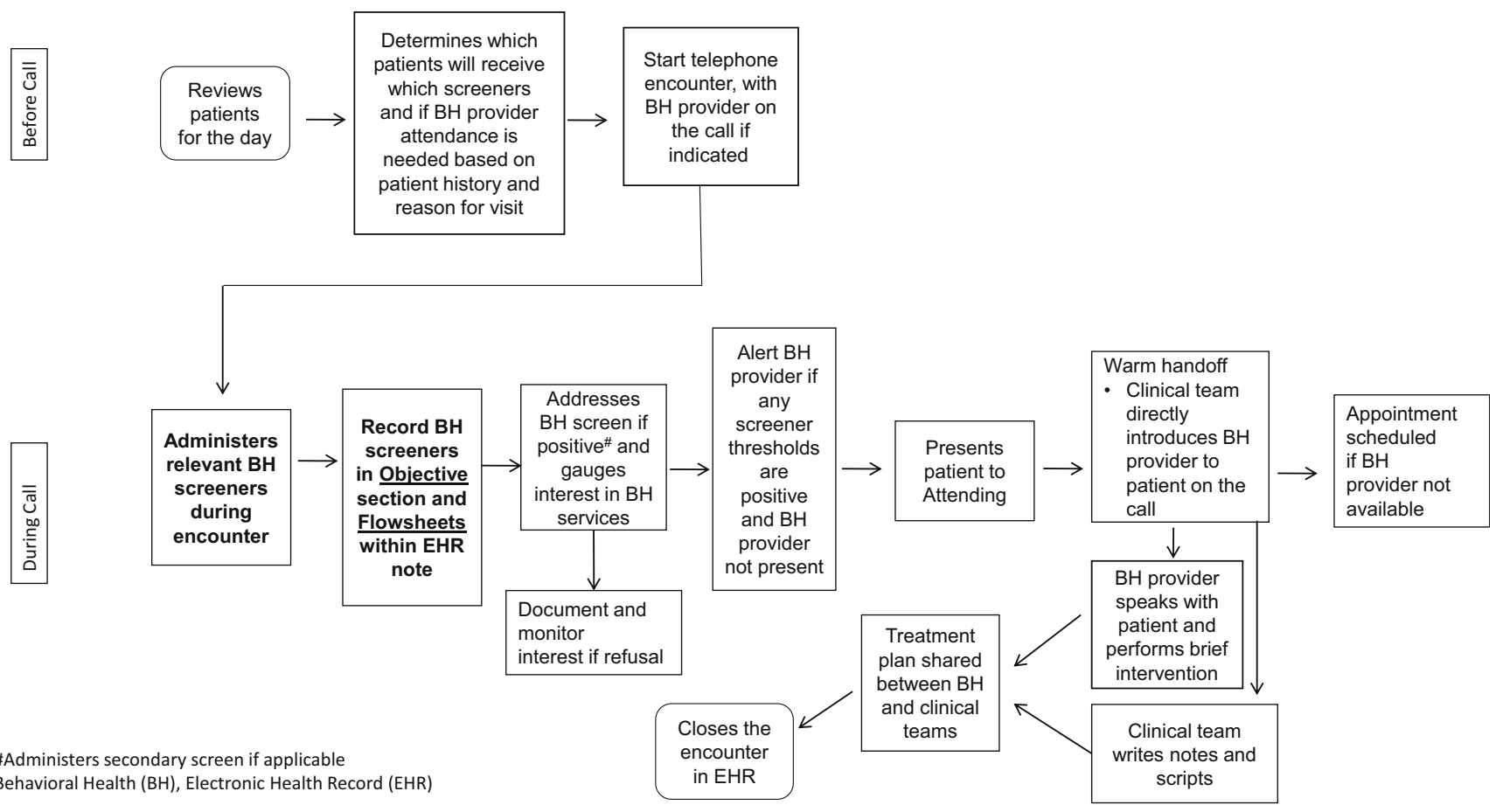

Fig. 1 Behavioral health integrated telehealth workflow 
concerns. In these cases, she would join the breakout room at the beginning of the visit. In situations where behavioral health topics arose in the breakout room, but were not identified either via the screeners or the behavioral health consultant's pre-review, the students returned to the main room and asked her to join the visit.

\section{Week 3}

Based on week 1 and 2 activities, the students developed a 20min behavioral health training. The training provided guidance on administering and interpreting the screeners within the clinic workflow, addressing behavioral health concerns presented during patient screening, and documenting the information collected during the screen. This training was presented in a meeting of all SOAR elective students who then completed a survey related to the training.

\section{Week 4}

During the final elective week, students compiled the training survey results. A total of 33 individuals completed the evaluation survey: 25 fourth-year medical students, 1 third-year medical student, 5 faculty, and 1 SOAR staff member. Nearly all participants reported feeling (1) comfortable/ extremely comfortable performing the behavioral health screeners after training (85 to $94 \%$ ), (2) confident/extremely confident to perform behavioral health activities following training (88 to $94 \%$ ), and (3) satisfied with the training content (97\%) and length (97\%). Open-ended responses indicated that the most helpful component of the training was (1) provision of charts/workflows as visual aids $(n=10)$, (2) explanation clarity $(n=4)$, and (3) detailed overview of the screeners $(n=$ 3 ). Areas for improvement included suggestions for a clinical example illustrating use of screeners and workflow $(n=4)$, more detailed screener instructions for junior students $(n=3)$, procedures for behavioral health emergencies $(n=1)$, as well as inclusion of screeners for other behavioral health concerns $(n=1)$. Based on the feedback provided by the trainees, the students revised the training materials and posted a final version to the university's online learning platform for future volunteer viewing.

\section{Implementation Results}

Three virtual clinics were held during the elective for a total of 20 patients. All 20 patients received PHQ-2 behavioral health screening. Twelve patients met with the behavioral health team for further screening either as result of positive screens or because they presented with symptoms or history. The following additional screeners were completed based on patient needs: PHQ-9 (7 patients), PHQ-4 (1 patient), and CAGE-
AID (3 patients). Based on screening results and prior history, nine patients were referred for a meeting with the behavioral health consultant, and seven received brief interventions (two declined). Primary diagnoses included depression and anxiety, and we referred three patients for external counseling and/or psychiatric services. External referrals were based on pre-existing diagnoses and history of ongoing services. No new psychiatric medication was prescribed during this time period; however, psychiatric medication was refilled for three patients. When medications are prescribed at SOAR, the prescription is called into the pharmacy by the students under the direction of the supervising physician. Capitalizing on the interdisciplinary collaboration among the medicine and pharmacy students and faculty who volunteer for SOAR, the clinic is able to use GoodRX to locate pharmacies that offer the medication as the lowest cost to the patient.

\section{Post Elective: Lessons Learned and Next Steps}

With the conclusion of the fourth-year elective, the remaining SOAR faculty and volunteer students faced unexpected challenges continuing the work started by the students. Specifically, there was difficulty ensuring workflow fidelity after the conclusion of the elective. Even with the prerecorded training, it has been difficult transitioning the behavioral health responsibilities to less experienced first- and second-year medical students. Further, new volunteers seemed unaware of specific cut-offs and workflow. To address this, during every morning huddle, the behavioral health consultant reviews the screeners and cut-offs and reasons for patient referral to the consultant. Similarly, the pre-recorded training is approximately $20 \mathrm{~min}$ long, which is lengthy for students who require training on multiple topics prior to volunteering. Consequently, we plan to create 5-min minitrainings with the crucial components to be viewed by all new volunteers. Students who wish to take a more active role related to behavioral health in the clinic will view the entire training. Finally, although we were in the process of implementing an evidence- and competency-based curriculum for telemedicine, given the urgency of the situation, the telehealth technology team had to rapidly change from inperson to a telehealth platform to provide uninterrupted service to our underserved patients while complying with local and state rules. Future work will continue to develop and implement competency-based telemedicine curriculum.

While sudden transitions due to COVID-19 resulted in many challenges, we also discovered important opportunities and insights. First, we realized that our fourth-year students provided a highly trained and motivated workforce and ready to help implement necessary changes. Consequently, modeled after the successful elective described here, we plan to develop future, on-going fourth-year elective rotations to be completed 
by one or two students at a time. These students will work on specific projects such as (1) incorporating behavioral health into the in-person SOAR clinic workflow, (2) performing quality assurance to track behavioral health activities and ensuring fidelity, and (3) helping implement medication-assisted treatment for SOAR patients who desire treatment of opioid use disorder. Additionally, the sudden transition to providing behavioral health via virtual clinics resulted in more private than anticipated settings for screening and referral. When SOAR clinic is conducted in-person, there may be up to five students per patient room, but virtual breakout sessions are limited to one senior student and one to two junior students. The challenge will be translating this approach back to inperson settings. Finally, even though our timeline for implementation of behavioral health using telehealth technology was suddenly compressed, implemented during a 2-week period rather than over 12 months, the crisis created by COVID19 provided an unexpected workforce to implement these changes at a time of incredible need. Consequently, this project resulted in rapid integration of virtual behavioral health services into a student-run free clinic, allowing us to better serve the healthcare needs of our community while providing a critical educational experience for our students.

Acknowledgments We would like to acknowledge Clementina Aiyudu and Urmil Patel for their work on the project and David Sperling and Christine Klein for their rapid re-organization of the fourth-year medicine clerkships.

Funding A 2019 HRSA Primary Care Training and Enhancement: Integrating Behavioral Health in Primary Care grant \#T0BHP33103 funded faculty and staff effort.

\section{Compliance with Ethical Standards}

Disclosure On behalf of all authors, the corresponding author states that there is no conflict of interest.

\section{References}

1. Bashshur R, Doarn CR, Frenk JM, Kvedar JC, Woolliscroft JO. Telemedicine and the COVID-19 pandemic, lessons for the future. Telemed e-Health. 2020;26(5):571-3.

2. Corruble E. A viewpoint from Paris on the COVID-19 pandemic: a necessary turn to telepsychiatry. J Clin Psychiatry. 2020;81(3): 20 com13361.

3. Liu S, Yang L, Zhang C, Xiang Y-T, Liu Z, Hu S, et al. Online mental health services in China during the COVID-19 outbreak. Lancet Psychiatry. 2020;7(4):e17-8.
4. Wright JH, Caudill R. Remote treatment delivery in response to the COVID-19 pandemic. Psychother Psychosom. 2020;89(3):130-2.

5. Zhou X, Snoswell CL, Harding LE, Bambling M, Edirippulige S, Bai X, et al. The role of telehealth in reducing the mental health burden from COVID-19. Telemed e-Health. 2020;26(4):377-9.

6. Ferrel MN, Ryan JJ. The impact of COVID-19 on medical education. Cureus. 2020;12(3):e7492.

7. Kanneganti A, Sia C-H, Ashokka B, Ooi SBS. Continuing medical education during a pandemic: an academic institution's experience. Postgrad Med J. 2020;96:384-6.

8. Mian A, Khan S. Medical education during pandemics: a UK perspective. BMC Med. 2020;18(1):100.

9. Newman NA, Lattouf OM. Coalition for medical education - a call to action: a proposition to adapt clinical medical education to meet the needs of students and other healthcare learners during COVID19. J Card Surg. 2020;35(6):1174-1175.

10. Rose S. Medical student education in the time of COVID-19. JAMA [Internet]. 2020 Mar 31 [cited 2020 May 27]; Available from: https://jamanetwork.com/journals/jama/fullarticle/2764138.

11. Butala NM, Murk W, Horwitz LI, Graber LK, Bridger L, Ellis P. What is the quality of preventive care provided in a student-run free clinic? J Health Care Poor Underserved. 2012;23(1):414-24.

12. Simpson SA, Long JA. Medical student-run health clinics: important contributors to patient care and medical education. J Gen Intern Med. 2007;22(3):352-6.

13. Swartz MK. The contributions of student-run free clinics. J Pediatr Health Care. 2012;26(6):397.

14. Khonsari N, Davis K, Wolf D. Implementing a psychiatry clinic in a student-run setting: a medical student perspective. Acad Psychiatry. 2019;43(3):340-3.

15. Knoll O, Chakravarthy R, Cockroft JD, Baddour N, Jordan S, Weaver E, et al. Addressing patients' mental health needs at a student-run free clinic. Community Ment Health J [Internet]. 2020 May 21 [cited 2020 May 27]; Available from: http://link.springer. com/10.1007/s10597-020-00634-3.

16. Ramos MA, Rotenstein LS, Mata DA. Student-run free clinics: the USA's psychiatry recruitment solution? Lancet Psychiatry. 2016;3(4):321-2.

17. Schweitzer PJ, Rice TR. The student-run clinic: a new opportunity for psychiatric education. Acad Psychiatry. 2012;36(3):233-6.

18. Soltani M, Smith S, Beck E, Johnson M. Universal depression screening, diagnosis, management, and outcomes at a student-run free clinic. Acad Psychiatry. 2015;39(3):259-66.

19. Substance Abuse and Mental Health Services Administration. SAMHSA-HRSA Center for Integrated Health Solutions (CIHS). [cited 2020 Jun 8]. Available from: https://www.samhsa.gov/ integrated-health-solutions.

20. Portage County Community Health Partners. 2019 Portage County Community Health Needs Assessment [Internet]. 2019 Dec [cited 2020 Jun 5]. Available from: https://www.co.portage.oh.us/sites/ portagecountyoh/files/pages/final_2019_portage_county_chna 12-6-19_0.pdf.

Publisher's Note Springer Nature remains neutral with regard to jurisdictional claims in published maps and institutional affiliations. 